\title{
Shore-based monitoring of flow dynamics in a steep bedrock canyon river
}

\author{
Saber Ansari ${ }^{1, *}$, Colin D. Rennie ${ }^{1}$, Jeremy G. Venditti ${ }^{2}$, Eva Kwoll ${ }^{3}$, Kirsti Fairweather ${ }^{2}$ \\ ${ }^{1}$ Civil Engineering, University of Ottawa, Ottawa, Ontario, Canada, K1N 6N5 \\ ${ }^{2}$ Department of Geography, Simon Fraser University, Burnaby, British Columbia, Canada V5A 1S6 \\ ${ }^{3}$ Department of Geography, University of Victoria, Victoria, British Columbia, Canada V8P 5C2
}

\begin{abstract}
The pace of landscape evolution is set by bedrock erosion in canyons. This phenomenon occurs by various geological processes including plucking of bedrock blocks and abrasion by saltating bedload and suspended load in highly turbulent flows. For a better understanding of the river flow characteristics in bedrock rivers, a comprehensive study of flow dynamics was undertaken in Black Canyon in the Fraser River, British Columbia. We used shore-based video imagery of the river to study surface flow dynamics. The shore-based monitoring system consisted of a Campbell Scientific camera mounted at the top of the canyon walls. We monitored the water surface boils due to upwelling and determined river surface flow velocities from the shore-based imagery. Automatic detection of the upwelling surface boils leads to a better understanding of the secondary circulation patterns and flow structures in this large steep river bedrock canyon. The data collection and analytical procedures developed in this research are cost-effective tools for remotely determining flow dynamics, which can be applied to other rivers.
\end{abstract}

\section{Introduction}

Surface processes significantly influence landscape. Interconnected dynamic interior and exterior earth processes affect surface landscape in long-term. Bedrock canyon erosion is amongst the most significant processes affecting landscape dynamics. Bedrock incision in rivers can be the result of plucking and abrasion processes. Understanding river hydraulics in bedrock canyons is crucial in the better quantification of incision processes. This can lead to the development of more robust sediment transport models in steep bedrock canyon conditions. Field observations and flow measurement is important in such studies and can be implemented in the calibration of the existing sediment models.

The advent of low cost, high-resolution digital cameras and also the fast development of different computer vision methods have led to the broad usage of image-based monitoring systems in earth sciences. Various studies have been conducted in which terrestrial monitoring was employed for efficient and effective data gathering (e.g. [1, 2, 3],). Development of different monitoring systems has also led to the application of these cost-

*Corresponding author : sansa048@uottawa.ca 
effective methods in quantified data collection e.g. [4]. Extensive application of the monitoring methods was also developed to the open channel flow measurement era. Particle Image Velocimetry (PIV) that was mostly used for laboratory flow measurements was later extended to a novel method which could perform large-scale flow measurement; Large-Scale Particle Image Velocimetry (LSPIV) was first introduced and employed by researchers such as [5] and [6]. Recent fast progress in computer vision techniques has led to innovation in this field and the application of cost-effective high-quality cameras in open channel flow measurements. Several studies have been conducted using various image analysis and computer vision techniques to improve the existing methods e. g. [7], [8], [9] and [10]. Most of these projects have focused on river discharge measurements using a series of consecutive images or recorded video files. The initial objective of the present study is to extract specific quantified data describing the flow field.

In a previous study on Fraser River by Venditti et. al. [11] it was found that as flow enters a bedrock canyon it plunges towards the bottom and the walls causing a velocity inversion. A lateral circulation then causes upwelling and eruption of surface boils along the canyon walls. The main focus of this study was the development of a monitoring system which could track and quantify these boils.

\section{Methodology}

\subsection{Study area}

A comprehensive study was conducted on the Black Canyon of the Fraser River. As the longest river in British Columbia, flowing for $1,375 \mathrm{~km}$ this river starts in the Rocky Mountains and discharges to the Strait of Georgia (Figure 1). The draining watershed of this river has an area of about $228,000 \mathrm{~km}^{2}$, and the mean discharge at the river mouth is about $3550 \mathrm{~m}^{3} / \mathrm{s}$. The Fraser River includes both alluvial and bedrock sections. Alluvial sections are mostly dominant in the river's length downstream of Hope. On the other hand, upstream of Hope the river mostly includes bedrock canyons [12]. The bedrock bound reaches, which include Black Canyon, comprise about $18 \%$ of the total length of the river [11].

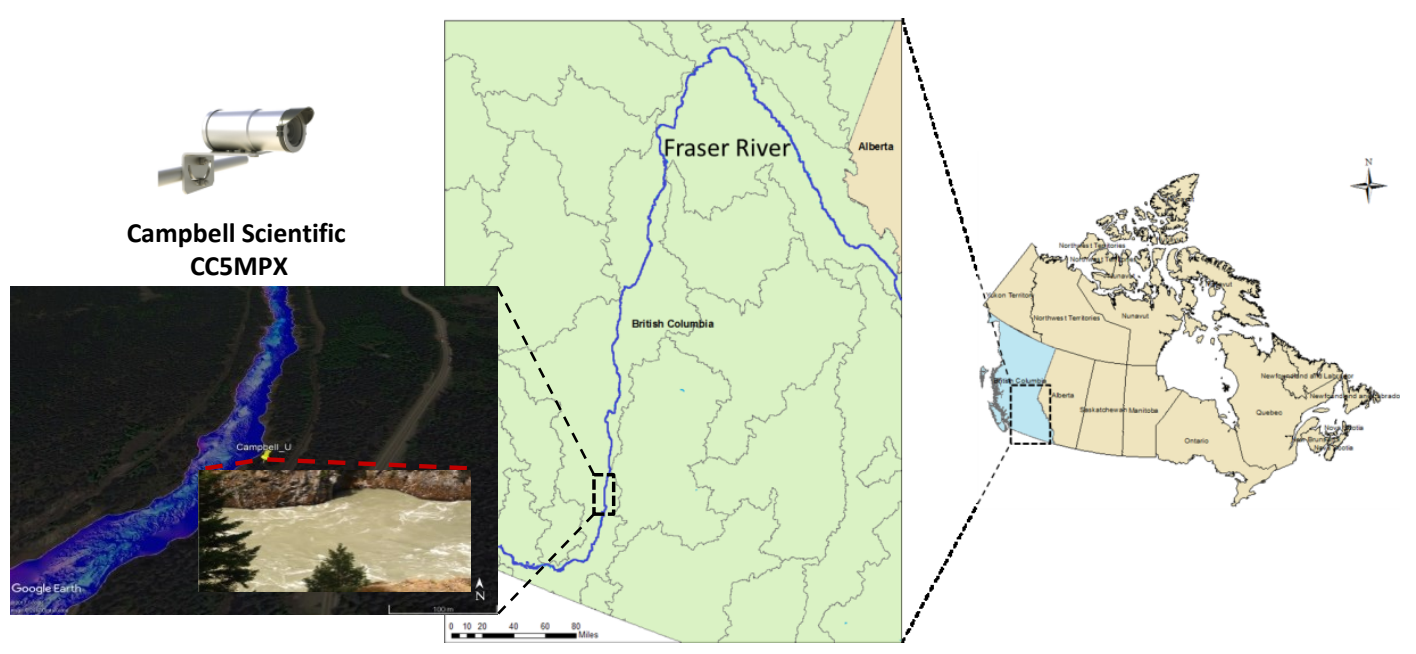

Fig. 1. Study area, Fraser River, Campbell Scientific CC5MPX camera and its field of view 


\subsection{Instrumentation}

A Campbell Scientific CC5MPX camera was mounted along the river above Black Canyon (Figure 1). The CC5MPX cameras are light high-resolution cameras that were programmed to capture still images and record one-minute video files during daylight. Specifications of the camera are presented in Table 1. Field of view (FOV) of the cameras is also shown in Figure 1. As shown in the figure the camera was mounted in a way to monitor the surface boils caused by upwelling in the river.

Table 1. Campbell Scientific CC5MPX specifications

\begin{tabular}{|l|l|}
\hline Diameter: $9.3 \mathrm{~cm}$ & Length: $22 \mathrm{~cm}$ \\
\hline Weight: $1.06 \mathrm{Kg}$ & Power: 9 to $16 \mathrm{Vdc}$ \\
\hline Operating temperature range: -40 to $+60^{\circ} \mathrm{C}$ & Standard lens: 4 to $12 \mathrm{~mm}\left(27^{\circ}\right.$ to $\left.80^{\circ} \mathrm{FOV}\right)$ \\
\hline Zoom lens: 10 to $40 \mathrm{~mm}\left(9^{\circ}\right.$ to $\left.35^{\circ} \mathrm{FOV}\right)$ & \\
\hline
\end{tabular}

\subsection{Video processing}

A video processing algorithm was developed to analyze the recorded video files. The main purpose of developing this algorithm was an automatic analysis of the video files to extract quantified results from water surface boils. Further analysis of the quantified results can lead to a better understanding of the secondary circulation patterns and flow structures in this large steep river bedrock canyon.

Figure 2 illustrates the flowchart of the developed video processing algorithm. This algorithm is divided into four main subsections; pre-processing, geo-rectification, detection and tracking surface boils, and real-world velocity estimation. Different subroutines of the developed video processing algorithm are explained here.

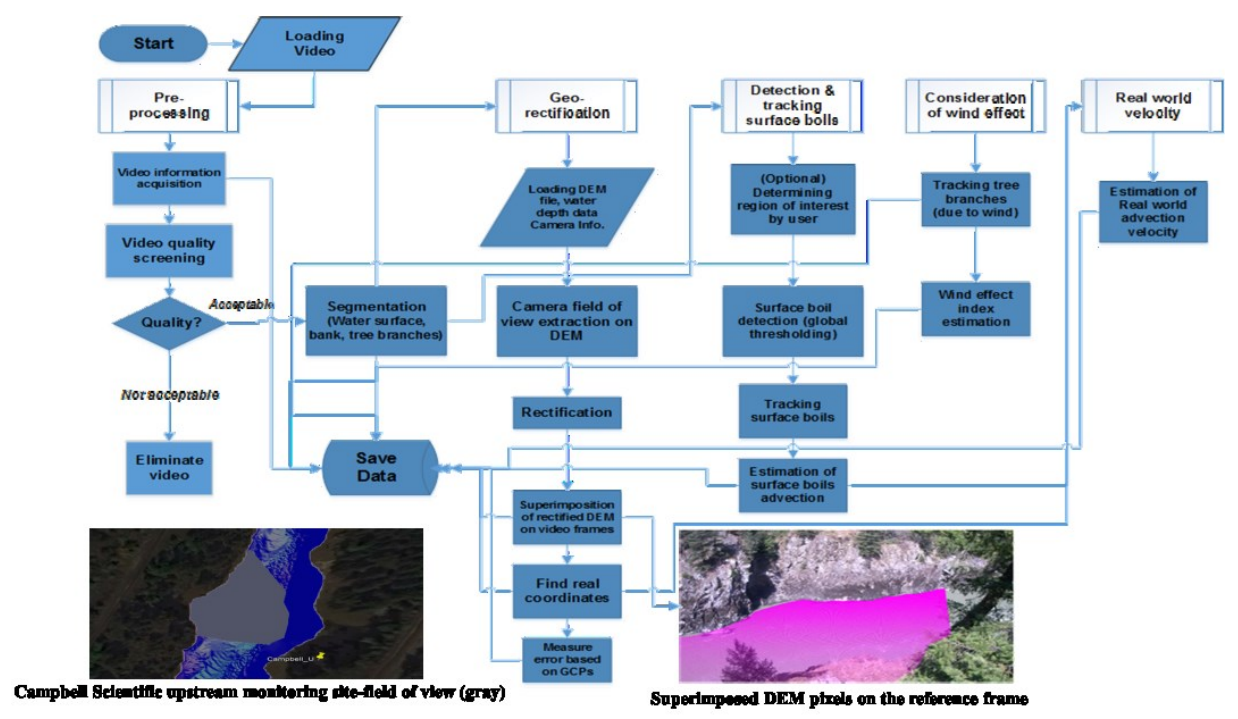

Fig. 2. Developed video processing algorithm in a pipeline. Figures on the bottom right and left show the superimposed camera FOV and the camera field of view (gray), respectively. 


\subsubsection{Pre-processing}

This step was developed to acquire basic information about the video files due to the large number of the recorded files. A basic video image quality classification was also included here to eliminate poor quality video files from the process of analysis.

\subsubsection{Geo-rectification}

Recorded frames had a $1280 \times 720$ pixels quality. A geo-rectification pipeline was utilized to calculate real-world coordinates and size of the pixels that differ throughout a single image. In this subroutine, a reference image that corresponds to the frames of the video files was rectified. As an essential step in the extraction of quantified data, the rectification algorithm assigns real-world coordinates to image pixels. The real world coordinates of the pixels are used in the final step to find the real world velocity of the surface boils. The detailed explanation of the employed image rectification method is presented in [3] and [4]. The digital elevation model (DEM) of the camera field of view was superimposed on the pixels of the reference image in order to assign real-world coordinates of the pixels (Figure. 2).

\subsubsection{Tracking surface boils}

Considering that the focus of this study was the surface boils induced by upwelling in the vicinity of the canyon walls, a global thresholding optimization method was first developed for surface boil detection. The threshold optimization method was developed using the intensity histogram of the video files with regard to the recording time of the video files. Once the video files were converted into binary frames, an optical flow method was used to track the detected surface boils.

The basics of the optical flow rely on the pixel intensity consistency change in a sequence of images. This led to the following equation known as flow constraint equation.

$$
\frac{\partial I}{\partial t}+\nabla I \cdot w=0
$$

In this equation $\nabla I=\left[I_{x}, I_{y}\right]^{T}$ is the spatial gradient of the image intensity function and $w=[u, v]^{T}$ represents the velocity function. Several methods have been presented to solve this equation. Amongst the several methods Horn Schunck [13] method is known to have the most reliable results[14]. They proposed to minimize the norm of the velocity gradient $\nabla w$ as regularization term [15]. In other words this method smoothens the results by considering that the neighbouring pixels have similar movements.

\subsubsection{Wind effect}

Wind can affect the detected intensity of the surface boils which may result in discrepancies in the extracted velocity estimations. Specifically, a wind effect occurred in which higher surface boil velocity magnitudes were observed during windy conditions, as compared to low wind conditions under similar discharge. Video files that displayed tree branch vibrations were corrected for this wind effect. In order to exclude this effect, in each set of video files for a given location with similar discharge, a reference video was selected that had the least movement of tree branches visible in the camera FOV. The difference between maximum surface boil velocity magnitude under a windy condition and the reference 
condition was used as a correction factor. Simply, boil surface velocity magnitudes under windy conditions were reduced by this correction factor.

\subsubsection{Real world velocity estimation}

The final subroutine was developed to convert the tracked surface boils to the real world velocity using the real world coordinates of the pixels in the image.

\section{Results and discussion}

The developed video processing algorithm was applied to a series of oblique shore-based recorded video files. Unlike other extant LSPIV methods, this algorithm was developed to mainly focus on a specific characteristic of the flow in the Black Canyon i.e. surface boils. Figure 3 illustrates a selected region of interest (ROI) of a section close to the right walls of the Black Canyon. Figure 3-B shows the same ROI at $\mathrm{T}=0 \mathrm{~s}$ and the second set of images (Figure 3-C) shows the same ROI after $0.5 \mathrm{~s}$.

Each set of pictures (Fig. $3 \mathrm{~B}$ and C) illustrate the selected region of interest. The second binary image is the result of tracked detected boils. Vectors on the binary images are the indication of the direction and magnitude of the velocity of the surface boil. The last figure in each set illustrates the estimated boil velocity.

A)
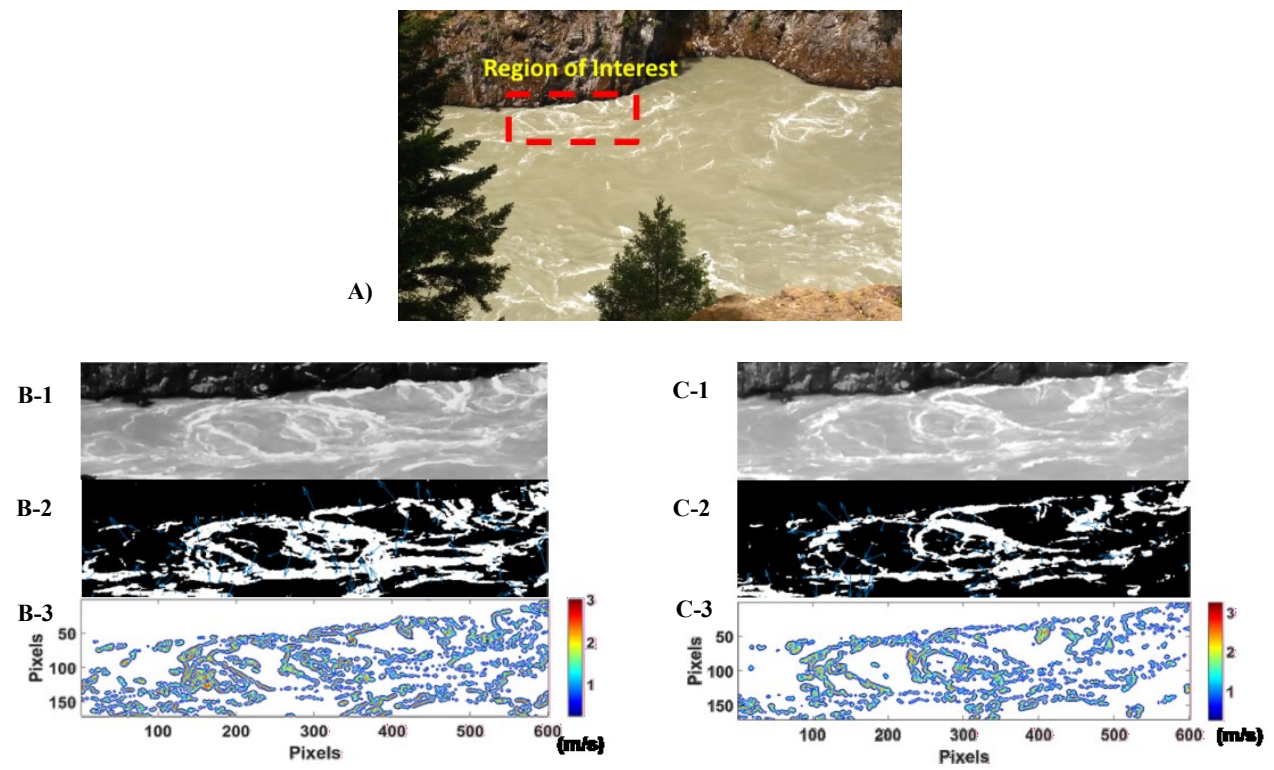

Fig. 3. Selected ROI including the detected surface boils and estimated surface boil velocity (m/s)-A) at $\mathrm{T}=0 \mathrm{Sec}$. B) at $\mathrm{T}=0.5 \mathrm{Sec}$.

Single eddies and their advection and advection velocity can be recognized in the pictures. Comparison of different frames allows for calculation of the velocity and the advection acceleration of the boils. The whole frame size could be analyzed using the developed method, however, focusing on a specific region of interest may lead to better analysis and better understanding of upwelling occurrence in the canyon. 


\section{Conclusion}

A shore-based monitoring method was proposed for a better understanding of the flow characteristics in a steep bedrock canyon. High-resolution cameras were utilized to record one-minute video files during high and low flow seasons. An automated video processing algorithm was developed to extract quantified results of the observed surface boils due to turbulent flow structure occurrences in the canyon. The surface boils can be detected and tracked using the developed method. Moreover, the rectification subroutine assigns correct real world coordinates to the pixels of the image. Results of this subroutine are used in the estimation of the real world velocity of the boils. An attempt was also made to exclude the wind effect in the surface boil velocity estimation. Further analysis of the results should be performed in low and high discharges for a better explanation of the turbulence flow structures in the steep bedrock canyon.

\section{References}

[1] D. Bourgault, "Shore-based photogrammetry of river ice," Can. J. Civ. Eng., vol. 35, pp. 8086, 2008.

[2] N. Chaouch, M. Temimi, P. Romanov, R. Cabrera, G. Mckillop, and R. Khanbilvardi, "An automated algorithm for river ice monitoring over the Susquehanna River using the MODIS data," Hydrol. Process., vol. 28, no. 1, pp. 62-73, 2014.

[3] S. Härer, M. Bernhardt, J. G. Corripio, and K. Schulz, "PRACTISE - Photo Rectification And ClassificaTIon SoftwarE (V.1.0)," Geosci. Model Dev, vol. 6, pp. 837-848, 2013.

[4] S. Ansari, C. D. Rennie, O. Seidou, J. Malenchak, and S. G. Zare, "Cold Regions Science and Technology Automated monitoring of river ice processes using shore-based imagery," Cold Reg. Sci. Technol., vol. 142, no. June, pp. 1-16, 2017.

[5] I. Fujita, M. Muste, and A. Kruger, "Large-scale particle image velocimetry for flow analysis in hydraulic engineering applications," J. Hydraul. Res., vol. 36, no. 3, pp. 397-414, 1998.

[6] I. Fujita and S. Aya, "Refinement of LSPIV technique for monitoring river surface flows," Build. Partnerships, vol. 16, pp. 1-9, 2008.

[7] R. J. Adrian, "Multi-point optical measurements of simultaneous vectors in unsteady flow-a review," Int. J. Heat Fluid Flow, vol. 7, no. 2, pp. 127-145, 1986.

[8] T. Vanden Berghe, "Image processing for a LSPIV application on a river," 2013.

[9] K. Flora, "Flood Flow Estimation using Large Scale Particle Image Velocimetry (LSPIV)," 2017.

[10] M. Jodeau, A. Hauet, J. L. E. Coz, Y. Bercovitz, F. Lebert, D. T. G. Edf, and U. R. Hhly, "Laboratory and field lspiv measurements of flow velocities using fudaa-lspiv, a free userfriendly software," Proc. 1st Int. Symp. Exhib. Hydro-Environment Sensors Softw. HydroSenSoft 2017, no. March, pp. 82-86, 2017.

[11] J. G. Venditti, C. D. Rennie, J. Bomhof, R. W. Bradley, M. Little, and M. Church, "Flow in bedrock canyons," Nature, vol. 513, no. 7519, pp. 534-537, 2014.

[12] D. Ham, "Morphodynamics and sediment transport in a wandering gravel-bed channel: Fraser River, British Columbia," Phd diss., 2005.

[13] B. K. P. Horn and B. G. Schunck, "Determining optical flow," Artif. Intell., vol. 17, no. 1-3, pp. 185-203, 1981.

[14] S. D. Thota, K. S. Vemulapalli, K. Chintalapati, and P. S. Srinivas, "Comparison Between The Optical Flow Computational Techniques,” vol. 4, no. 10, pp. 4507-4511, 2013.

[15] M. Khalid, L. Pénard, E. Mémin, M. Khalid, L. Pénard, and E. Mémin, "Application of optical flow for river velocimetry," in IGARSS 2017 -37th IEEE Geoscience and Remote Sensing Symposium, 2017, pp. 1-4. 